\title{
Sequencing of NOTCH1, GATA5, TGFBR1 and TGFBR2 genes in familial cases of bicuspid aortic valve
}

\author{
Ilenia Foffa ${ }^{1}$, Lamia Ait Alì , Paola Panesi ${ }^{1}$, Massimiliano Mariani ${ }^{2}$, Pierluigi Festa ${ }^{2}$, Nicoletta Botto ${ }^{2}$,
} Cecilia Vecoli ${ }^{1}$ and Maria Grazia Andreassi ${ }^{1 *}$

\begin{abstract}
Background: The purpose of our study was to investigate the potential contribution of germline mutations in NOTCH1, GATA5 and TGFBR1 and TGFBR2 genes in a cohort of Italian patients with familial Bicuspid Aortic Valve (BAV).

Methods: All the coding exons including adjacent intronic as well as $5^{\prime}$ and $3^{\prime}$ untranslated (UTR) sequences of NOTCH1, GATA5, TGFBR1 and TGFBR2 genes were screened by direct gene sequencing in 11 index patients (8 males; age $=42 \pm 19$ years) with familial BAV defined as two or more affected members.

Results: Two novel mutations, a missense and a nonsense mutation (Exon 5, p.P284L; Exon 26, p.Y1619X), were found in the NOTCH1 gene in two unrelated families. The mutations segregated with the disease in these families, and they were not found on 200 unrelated chromosomes from ethnically matched controls. No pathogenetic mutation was identified in GATA5, TGFBR1 and TGFBR2 genes.

Conclusions: Two novel NOTCH1 mutations were identified in two Italian families with BAV, highlighting the role of a NOTCH1 signaling pathway in BAV and its aortic complications. These findings are of relevance for genetic counseling and clinical care of families presenting with BAV. Future studies are needed in order to unravel the still largely unknown genetics of BAV.
\end{abstract}

Keywords: Bicuspid aortic valve, Direct gene sequencing, Genes

\section{Background}

Bicuspid aortic valve (BAV) is the most common congenital cardiac malformation, with a prevalence estimated between $0.5 \%$ and $2 \%[1,2]$. Nearly all patients with BAV develop serious aortic complications, including significant valve regurgitation, severe aortic stenosis, aortic aneurysm and dissection [3,4]. Therefore, the burden of BAV disease is more significant than any other congenital cardiac abnormalities [4]. Clinical studies have reported high heritability in families of patients with BAV [5-7], suggesting a Mendelian pattern of inheritance and the need for echocardiography screening in first-degree relatives of patients with BAV [8].

\footnotetext{
* Correspondence: andreassi@ifc.cnr.it

${ }^{1}$ CNR Istituto di Fisiologia Clinica, Via Moruzzi 1, Pisa 56124, Italy

Full list of author information is available at the end of the article
}

A family-based linkage analysis has identified genomic regions on chromosomes $18 \mathrm{q}, 5 \mathrm{q}$ and $13 \mathrm{q}$ responsible for BAV and associated cardiac malformations, but the precise genes within these regions were not defined [9].

To date, only mutations in the NOTCH1 gene, a signaling transmembrane receptor (2,556 amino acids) involved in multiple cell aspects of vascular development, have been associated with dominantly inherited BAV in a small number of families [10].

However, the rate of NOTCH1 gene mutations in familial forms of BAV and their association with different clinical complications remains incomplete [11,12]. Interestingly, McBride et al. demonstrated that mutations in the gene NOTCH1 are found in cases of left ventricular outflow tract (LVOT) malformations including aortic valve stenosis, coarctation of the aorta and hypoplastic left heart syndrome [13]. In particular, a much higher
C Biomed Central 
rate of bicuspid aortic valve (BAV) has been found in families with LVOT malformations compared to the general population, suggesting that BAV may be a forme frusta of the more serious LVOT malformations [13].

Recently, a missense mutation in transforming growth factor-beta receptor 2 gene (TGFBR2) was found in a patient with BAV and aortic aneurysm [14], but earlier studies found no mutation in either TGFBR1 or TGFBR2 in patients with familial and sporadic BAV disease $[15,16]$.

Finally, GATA5 was reported to have an essential role in cardiac morphogenesis and in aortic valve development $[17,18]$, and targeted deletion of this gene in mice leads to partially penetrant BAV [19]. Accordingly, a very recent study investigated the relationship between GATA5 gene variations and BAV disease identifying rare non-synonymous variations in the functionally important GATA5 transcriptional activation domain [20]. Conversely from NOTCH1 gene, the involvement of GATA5 has not been described in other LVOT malformations.

However, further studies are needed to assess the involvement of NOTCH1, GATA5 and TGFBR1 and
TGFBR2 mutations in patients with BAV and its associated aortic complications.

The purpose of our study was to investigate the potential contribution of germline mutations in NOTCH1, GATA5, TGFBR1 and TGFBR2 genes in a cohort of Italian patients with familial BAV.

\section{Methods}

\section{Study population}

A cohort of 11 unrelated Italian patients ( 8 males; age = $42 \pm 19$ years) with familial BAV were recruited for this study. Patients with sporadic BAV and/or with coexisting syndromes, such as Marfan syndrome, Turner syndrome and Di George syndrome or unknown syndromes were excluded from the study. Clinical evaluation consisted of medical history, physical examination, 2-dimensional echocardiography and a complete Magnetic Resonance Imaging (MRI) study. A detailed family history was obtained for each proband. Familial BAV was defined if two or more affected relatives had proven BAV diagnosed by echocardiography or cardiac magnetic resonance. Figure 1 shows the pedigree of BAV patients. Patient's

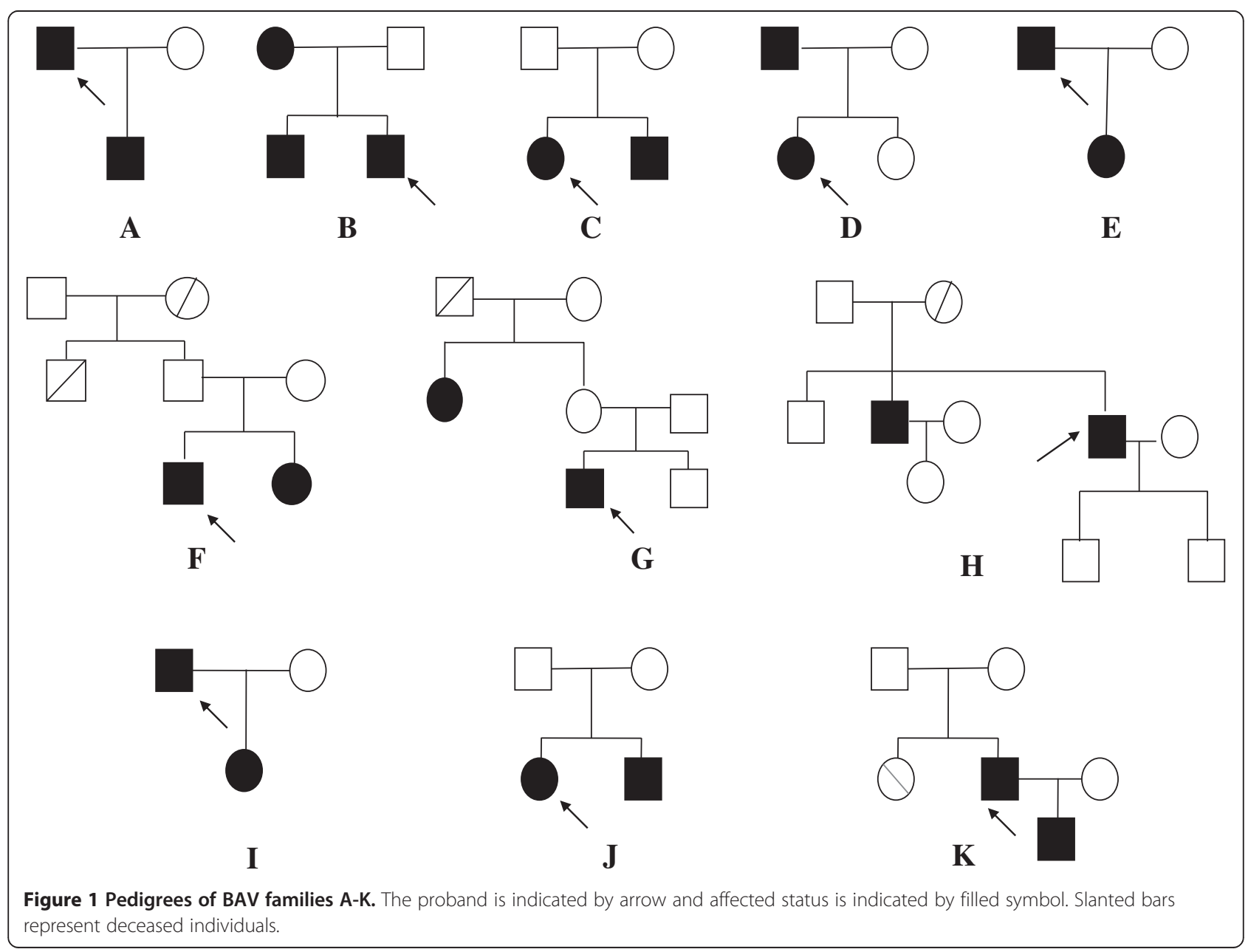


phenotype is reported in Table 1 . Two of the probands (18\%) had BAV with unknown morphology. Of the 9 probands with known BAV morphology, 7 (63\%) had fusion of the right/left coronary commissure whereas 2 (18\%) had a valve with anterior-posterior sinus without raphe. We also included a control cohort comprised of 100 ethnically unrelated individuals (60 male; age $=35 \pm 16$ years) without BAV as shown by echocardiography. This study was conducted with informed consent of every participant subject and was approved by the local Ethical Research Committee.

\section{Mutational sequencing analysis}

A mutational screening was performed by direct gene sequencing of all coding exons including adjacent intronic as well as $5^{\prime}$ and $3^{\prime}$ untranslated sequences of NOTCH1, GATA5 and TGFBR1, TGFBR2 genes. Genomic DNA was extracted from peripheral blood cells using the QIAGEN BioRobot ${ }^{\circledR}$ EZ1 System. Exons and the flanking intronic sequences were amplified by polymerase chain reaction (PCR) using specific primers as described previously for NOTCH1 and TGFBR1 and TGFBR2 [11,21] and using Primer3 (v. 0.4.0) software (http://frodo.wi.mit.edu/primer3/) based on the cDNA sequences available in GenBank for GATA5 screening.

Approximately, PCR amplifications were performed with a volume of 50 microl mixture containing $1.5 \mathrm{mM}$ $\mathrm{MgCl} 2,10$ mMdNTP, 50 ng genomic DNA, 20 micromol of each primer and $2.5 \mathrm{U}$ Taq DNA polymerase. PCR product was used for PCR sequencing reaction with the CEQ DTCS Quick Start Kit. The sequencing reaction products were purified by precipitation with ethanol, re-suspended in a sample loading solution and analyzed with a CEQ 8800 capillary sequence (Beckman Coulter, Germany) according to the manufacturer's protocol. Resulting sequences were analyzed using CEQ 8800 software packages and aligned against a reference sequence obtained from GenBank.

For prediction of the functional consequences of mutations on protein sequence, we used software programs available on the internet, namely PolyPhen-2 (http://genetics.bwh.harvard.edu/pph/) SIFT (http://sift. jcvi.org/) and Mutation Taster (www.mutationtaster. org/). PolyPhen-2 predicts the effect of an amino acid substitution on the structure and function of a protein, using sequence homology. The PolyPhen-2 score represents the probability that a substitution is damaging, so values nearer 1 are more confidently predicted to be deleterious (note that this is the opposite of SIFT).

SIFT uses sequence homology to predict whether an amino acid substitution will affect the protein function. SIFT score $<0.05$ indicates the amino acid substitution is damaging while scores $\geq 0.05$ are predicted to be tolerant. Mutation Taster is a free, web-based application for rapid evaluation of the disease-causing potential of DNA sequence alterations. Mutation Taster integrates information from different biomedical databases and uses established analysis tools. Analyses comprise evolutionary conservation, splice-site changes, loss of protein features and changes that might affect the amount of mRNA.

Detected mutations in probands were confirmed by sequencing in the opposite direction from another two independent PCR products.

The presence of a novel mutation was assessed in 200 ethnically reference alleles derived from volunteers by PCR-based restriction fragment length polymorphism (PCR-RFLP) or by direct gene sequencing.

\section{Results}

Clinical and demographic characteristics of each patient are summarized in Table 1. The group comprised $73 \%$

Table 1 Clinical and demographic characteristics of study population

\begin{tabular}{|c|c|c|c|c|c|}
\hline Proband & Sex & Age & Diagnosis & Phenotype & Other cardiac malformation \\
\hline A & M & 40 & $A S$ and $A A D$ & AP & / \\
\hline B & M & 25 & $A R$ and $A A D$ & $R-L$ & / \\
\hline $\mathrm{C}$ & $\mathrm{F}$ & 40 & $A R$ & unk & / \\
\hline D & $\mathrm{F}$ & 21 & $A R$ & $R-L$ & $\mathrm{COA}$ \\
\hline E & M & 37 & BAV & $R-L$ & / \\
\hline$F$ & M & 74 & $A S$ and $A A D$ & unk & / \\
\hline G & M & 17 & $\mathrm{Al}$ and $\mathrm{AAD}$ & $R-L$ & / \\
\hline $\mathbf{H}$ & M & 67 & $A R$ and $A A D$ & $R-L$ & / \\
\hline I & M & 38 & AS & $R-L$ & $\mathrm{COA}$ \\
\hline$J$ & $\mathrm{~F}$ & 65 & $A S$ and $A A D$ & AP & / \\
\hline K & $M$ & 37 & $A R$ and $A A D$ & $R-L$ & / \\
\hline
\end{tabular}

AS: aortic stenosis; AAD: Ascending Aortic Dilation; AR: Aortic regurgitation; Al: aortic insufficiency; CoA: Aortic Coarctation; AP: anterior-posterior no raphe; R-L; right/left coronary commissure; unk: unknown. 
males, and coarctation of the aorta $(\mathrm{CoA})$ as coexistent cardiovascular anomalies occurred in $18 \%$ of the cohort.

\section{Genetic analysis of $\mathrm{NOTCH} 1$ gene}

A mutational screening was performed by direct gene sequencing of all 34 coding exons including adjacent intronic as well as $5^{\prime}$ and $3^{\prime}$ untranslated sequences of NOTCH1 gene. Seventeen separate NOTCH1 sequence variants listed previously in public dbSNPs or in the literature were found in our patients, while five variants were novel. These included one synonymous variant (Exon 21, p.G1166G), two intronic (IVS11 + 63; IVS32-41) and two novel mutations (Exon 5,p.P284L; Exon 26, p.Y1619X). Details of the specific sequence variants and number of positive patients are summarized in Table 2 .

The novel $\mathrm{C}>\mathrm{T}$ heterozygous substitution in exon 5, resulting in the substitution of a Proline with a Leucine (P284L) at position 284, was found in the sequencing electropherograms of a 40-year-old male patient (Proband A; Figure 2). The identified missense mutation was absent in 200 ethnically reference alleles derived from volunteers. PolyPhen-2 analysis predicted the p.P284L mutation as probably damaging with a score of 0.993 . This result was confirmed by SIFT (score: 0.04 ).

The missense mutation (p.P284L) identified in our proband, tested by Mutation Taster, induced the loss of an important residue with putative significance for calcium binding to epidermal growth factor (EGF)-like 7 domain.

The proband, at the age of 39 years, presented severe calcified aortic valve and normal diameter of ascending aorta $(39 \mathrm{~mm})$. One year later, the patient underwent elective aortic valve and ascending aorta repair $(48 \mathrm{~mm})$ replacement. Family history and echocardiography analysis revealed the presence of BAV in his 7-year-old son. Genetic screening of the proband's child confirmed the presence of p.P284L mutation in exon 5 of NOTCH1 gene.

Moreover, we detected a novel heterozygous C-to-G transition, in exon 26, of nucleotide 4856 leading to a premature stop codon at position 1619 (p.Y1619X) instead of tyrosine in the sequencing electropherograms of

Table 2 Genetic variants within the human NOTCH1 gene identified in familial BAV patients

\begin{tabular}{|c|c|c|c|c|c|}
\hline $\begin{array}{l}\text { Genomic position } \\
\text { (NG_007458.1) }\end{array}$ & $\begin{array}{c}\text { Position } \mathrm{NOTCH} 1 \\
\text { gene }\end{array}$ & $\begin{array}{l}\text { cDNA position } \\
\text { (NM_017617.3) }\end{array}$ & $\begin{array}{l}\text { Protein } \\
\text { position }\end{array}$ & $\begin{array}{l}\text { Number of } \\
\text { patients }\end{array}$ & $\begin{array}{l}\text { rsSNP number } \\
\text { (dbSNP) }\end{array}$ \\
\hline g.26979 T > C & Exon 3 & c.312 T>C & p.N104N & 6 & rs4489420 \\
\hline g.31330 C > T & Exon 5 & c. $851 C>T$ & p.P284L & 1 & New \\
\hline g.31331 G > A & Exon 5 & c. $852 \mathrm{G}>\mathrm{A}$ & p.P284P & 4 & rs2229975 \\
\hline g.34650 T > C & IVS 9-43 & & & 3 & rs4880099 \\
\hline g. $33525 A>G$ & IVS $9+10$ & & & 2 & rs11145767 \\
\hline g.33601 C > A/T & IVS $9+86$ & & & 2 & rs113341997 \\
\hline g. $33617 C>T$ & IVS $9+102$ & & & 1 & rs10781498 \\
\hline g.33620C $>T$ & IVS $9+105$ & & & 1 & rs11574887 \\
\hline g. $34772 C>T$ & Exon 10 & c. 1635 C> T & p.D545D & 1 & rs11574889 \\
\hline g. $35062 A>G$ & IVS 11-9 & & & 3 & rs3124603 \\
\hline g.35367 G > A & IVS $11+63$ & & & 1 & New \\
\hline g.35591C > T & IVS $12+94$ & & & 1 & rs62579232 \\
\hline g. $36347 G>A$ & IVS $13+70$ & & & 2 & rs3812609 \\
\hline g.37307 T > C & Exon 14 & c. $2265 \mathrm{~T}>\mathrm{C}$ & p.N755N & 4 & rs2229971 \\
\hline g.39978G > A & IVS 16-4 & & & 3 & rs3125001 \\
\hline g.40058C > T & Exon 17 & c. 2664 C > T & p.H888H & 1 & rs61751548 \\
\hline g.40085C > T & Exon 17 & c. 2691 C > T & p.A897A & 1 & rs11574895 \\
\hline g. 42820 C > T & Exon 21 & c. 3498 C > T & p.G1166G & 1 & New \\
\hline g. 45953 C > G & Exon 26 & c. $4856 C>$ G & p.Y1619X & 1 & New \\
\hline g. $47532 C>T$ & Exon 27 & c. 5094 C > T & p.D1698D & 3 & rs10521 \\
\hline g.48831 T > C & IVS $30-43$ & & & 2 & rs3124594 \\
\hline g.49928C > T & IVS $30-12$ & & & 1 & rs11574908 \\
\hline g.51487 C > T & IVS $32-41$ & & & 1 & New \\
\hline
\end{tabular}




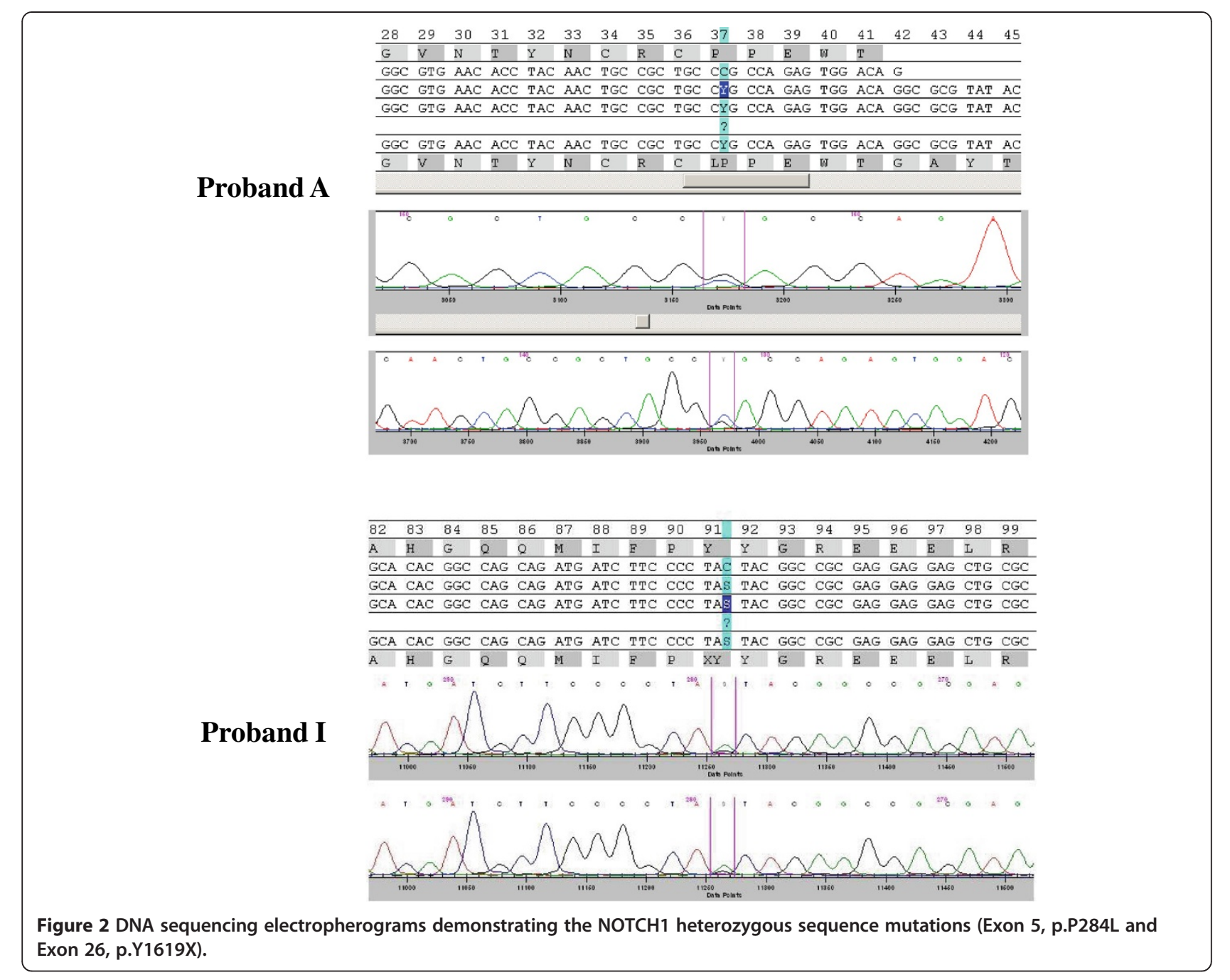

a 38-year-old male patient (Proband I; Figure 2). The identified mutation was absent in 200 ethnically reference alleles derived from volunteers. At age 6 months the proband had surgery to correct the CoA, while at age 25 years he underwent elective aortic valve replacement. Family history and echocardiography analysis revealed the presence of BAV in his 5-year-old daughter. Genetic screening of the proband's child confirmed the presence of p.Y1619X mutation in exon 26 of NOTCH1 gene.

Table 3 Genetic variants within the human GATA5 gene identified in familial BAV patients

\begin{tabular}{|c|c|c|c|c|}
\hline Position GATA5 gene & HGVS names & Protein position & Number of patients & rsSNP number (dbSNP) \\
\hline Exon1 & C.199 A > C & p.Thr67Pro & 5 & rs6142775 \\
\hline Exon 2 & c. 609 C > T & p.Asp203Asp & 7 & rs41305803 \\
\hline Exon 2 & c. 678 C > T & p.Leu226Leu & 1 & rs139428354 \\
\hline IVS 4-14 & c.826-14 G > A & & 1 & rs140408446 \\
\hline Exon 4 & c. $852 \mathrm{G}>\mathrm{A}$ & p.Lys284Lys & 8 & rs6587239 \\
\hline IVS 5-16 & c.914-16 C > T & & 1 & New \\
\hline Exon 5 & C. $981 \mathrm{G}>\mathrm{C}$ & p.Ser327Ser & 7 & rs6061243 \\
\hline IVS 6-78 & c. $1039-78$ C > G & & 5 & rs113912772 \\
\hline Exon6 & c. $1128 A>G$ & p.Pro376Pro & 11 & rs 6061550 \\
\hline
\end{tabular}




\section{Genetic analysis of GATA5 gene}

Sequencing of all 6 exons and splice signal sequences of GATA5 in our cohort revealed five synonymous, one non-synonymous and three intronic variants. Details of the specific sequence variants and number of positive patients are summarized in Table 3. We identified one novel intronic variant ( $\mathrm{C}>\mathrm{T}$; IVS $5-15$ ) in one patient, but it was also present in our control group.

\section{Genetic analysis of TGFBR1 and TGFBR2 genes}

TGFBR1 and TGFBR2 genes were analyzed by direct gene sequencing of all coding exons including adjacent intronic as well as $5^{\prime}$ and $3^{\prime}$ untranslated sequences. No pathogenetic mutation was identified; we found only known common genetic polymorphisms. Details of the specific sequence variants in TGFBR1 and TGFBR2 genes are summarized in Table 4.

\section{Discussion}

In our cohort of 11 familial patients with BAV two novel mutations were found in the NOTCH1 gene in two unrelated families. The mutations segregated with the disease in these families, and they were not found in 200 chromosomes from ethnically matched controls.

On the contrary, analysis of GATA5, TGFBR1 and TGFBR2 genes identified only known genetic variants. Our findings confirm the important role of NOTCH1 as conserved intracellular regulator in the pathogenesis of congenital valve disease and in its complications. Indeed, the finding of two independent de novo mutations in this gene in a small number of affected subjects is compelling evidence of disease causation.

NOTCH1 encodes for a transmembrane protein that activates a signaling pathway with a major role in cardiac embryogenesis, including aortic and pulmonary valve development as well as the development and maintenance of the aorta and other great vessels [22]. Murine model studies indicate that Notch signaling promotes the epithelial-to mesenchyme transition that gives rise to the primordial cardiac valve, which later is sculpted into mature valves [23]. Moreover, a recent study showed that heterozygous Notch1-null (Notch1(+/)(-) mice had greater than fivefold more aortic valve calcification than age- and sex-matched wild type littermates [24].

Indeed, in vitro studies showed that Notch1 signaling repressed valvular Bmp2 expression, and de-repression of Bmp2 was involved in calcification induced by Notch1 inhibition. Thus, Notch1 signaling appears to prevent aortic valve calcification in part by repressing Bmp2 expression within the valve [24].

Defective NOTCH signaling might also contribute to aortic valve calcification by repressing the activity of the hairy-related family of transcription factors (Hrt), and thereby also repressing the Runx $2 / \mathrm{Cbfa} 1$ pathway, a central transcriptional regulator of osteoblast cell fate [24,25].

In addition, there is increasing evidence of interaction between the Notch1 pathway and transforming growth factor beta (TGF $\beta$ ) [26]. This is particularly important in light of evidence showing that TGF $\beta$ plays an important role in vascular remodeling and alterations in TGF $\beta$ signaling activity might enhance matrix degradation $[27,28]$.

In 2005, the discovery of a nonsense (p.R1108X) and a frameshift mutation (p.H1505del) in two families represented the first demonstration of NOTCH1 germline mutations as a cause of BAV and severe valve calcification in non-syndromic autosomal-dominant human pedigrees [10].

Subsequently, two novel mutations (p.T596M and p. $\mathrm{P} 1797 \mathrm{H})$ located in highly conserved regions of the protein were identified in patients with sporadic BAV, providing evidence that mutations in NOTCH1 do not only play a role in familial BAV, but can also be observed in approximately $4 \%$ of sporadic cases [11]. Furthermore, a targeted mutational analysis of NOTCH1 demonstrated the presence of two novel mutations (p.A1343V and p. P1390T) among patients with BAV with sporadic and concomitant thoracic aortic aneurysms, providing evidence that mutations in NOTCH1 may also confer susceptibility to aortic aneurysm formation [12].

In a very recent paper, Kent et al. suggested a NOTCH1-dependent mechanism that produces stenotic, insufficient and/or calcified aortic valves with a rare aneurysm, and a NOTCH1-independent mechanism that

Table 4 Genetic variants within the human TGFBR1 and TGFBR2 genes identified in familial BAV patients

\begin{tabular}{cccccc}
\hline Gene & Position TGFBR1 TGFBR2 gene & HGVS names & Protein position & Number of patients & rsSNP number \\
\hline TGFBR1 & Exon 1 & c.68_76delCGGCGGCG & p.Ala23Ala & 4 & rs11466445 \\
& IVS 7 + 24 & c.1255+24 G > A & & 2 & rs334354 \\
TGFBR2 & IVS 3 + 7 & c.338 + 7 A > G & & 2 & rs1155705 \\
& IVS 5-4 & c.455-4 T > A & & 3 & rs11466512 \\
& Exon 4 & c.458delA & p.Lys153 & 8 & rs79375991 \\
\hline
\end{tabular}


produces highly penetrant ascending aortic aneurysm (AscAA) in the presence of a non-calcified and often normally functioning BAV [29]. Our data appear to agree with and fit well into the proposal made in this article by Kent et al., since we found two novel mutations in these patients with valve malformation, calcification, and dysfunction as predominant phenotype caused by altered NOTCH1 signaling.

Conversely, we did not identify any pathogenetic mutations in GATA5. The non-synonymous variant Thr67Pro located in the transcriptional activator domain encoded by exon 1, detected with a frequency of $45 \%$ in our population, is a common genetic polymorphism previously described $[20,30]$.

Regarding to TGFBR1 and TGFBR2 genes, in contrast with a recent paper showing a missense mutation in TGFBR2 gene in a patient with BAV and aortic aneurysm [14], our findings support the studies suggesting that mutations in these genes are rarely identified in patients with familial BAV $[15,16]$.

\section{Conclusion}

Although the small number of patients (11) might be an important study limitation, we have identified two novel NOTCH1 mutations in two Italian families with BAV. Our findings confirm the critical role of NOCTH1 in the development of aortic valve and aortic complications. Genetic screening for NOTCH1 mutation may be considered for diagnosis and family screening as part of the standard management of patients with family history of BAV. However, the etiology of this common valve disease is largely unknown. Future studies based on new genetic screening strategies, the so-called nextgeneration sequencing, are needed in order to unravel the still largely unknown genetics of BAV.

Abbreviations: $\mathrm{BAV}=$ Bicuspid Aortic Valve, TGFBR1 $=$ transforming growth factor-beta receptorI gene; $T G F B R 2=$ transforming growth factor-beta receptorII gene; PCRRFLP $=$ PCR-based restriction fragment length polymorphism; $\mathrm{MAF}=$ minor allele frequency; $\mathrm{CoA}=$ coarctation of the aorta; TGF $\beta=$ transforming growth factor beta; $\mathrm{MRI}=$ Magnetic Resonance Imaging; AscAA = ascending aortic aneurysm; LVOT $=$ left ventricular outflow tract.

\section{Competing interests}

The authors declare that they have no competing interests.

\section{Authors' contributions}

IF participated in the design of the study, carried out the molecular genetic studies, performed the statistical analysis and drafted the manuscript. MGA conceived the study, participated in its design and coordination and critically revised the manuscript. PP carried out the molecular genetic studies. MM, AAL and PF participated in design of the study and in the enrollment of the patients. NB and CV performed the genetic analysis and critically revised the manuscript. All authors read and approved the final manuscript.

\section{Acknowledgments}

We are indebted with Ms. Alison Frank for her invaluable help in editing the manuscript.

\section{Author details}

${ }^{1}$ CNR Istituto di Fisiologia Clinica, Via Moruzzi 1, Pisa 56124, Italy. ${ }^{2}$ Fondazione CNR-Regione Toscana Gabriele Monasterio, Pisa, Italy.

Received: 31 October 2012 Accepted: 2 April 2013

Published: 11 April 2013

\section{References}

1. Mordi I, Tzemos N: Bicuspid aortic valve disease: a comprehensive review. Cardiol Res Pract 2012, 2012:196037

2. Laforest B, Nemer M: Genetic insights into bicuspid aortic valve formation. Cardiol Res Pract 2012, 2012:180297.

3. Michelena HI, Khanna AD, Mahoney D, Margaryan E, Topilsky Y, Suri RM, Eidem B, Edwards WD, Sundt TM 3rd, Enriquez-Sarano M: Incidence of aortic complications in patients with bicuspid aortic valves. JAMA 2011, 306:1104-1112.

4. Lewin MB, Otto CM: The bicuspid aortic valve: adverse outcomes from infancy to old age. Circulation 2005, 111:832-834

5. Huntington $\mathrm{K}$, Hunter $\mathrm{AG}$, Chan $\mathrm{KL}$ : A prospective study to assess the frequency of familial clustering of congenital bicuspid aortic valve. J Am Coll Cardiol 1997, 30:1809-1812.

6. Cripe L, Andelfinger G, Martin J, Shooner K, Benson DW: Bicuspid aortic valve is heritable. J Am Coll Cardiol 2004, 44:138-143.

7. Glick BN, Roberts WC: Congenitally bicuspid aortic valve in multiple family members. Am J Cardiol 1994, 73:400-404

8. Warnes CA, Williams RG, Bashore TM: ACC/AHA 2008 guidelines for the management of adults with congenital heart disease: a report of the American College of Cardiology/American Heart Association Task Force on Practice Guidelines (Writing committee to develop guidelines on the management of adults with congenital heart disease). J Am Coll Cardiol 2008, 52:e1-e121.

9. Martin LJ, Ramachandran V, Cripe LH, Hinton RB, Andelfinger G, Tabangin M, Shooner K, Keddache M, Benson DW: Evidence in favor of linkage to human chromosomal regions $18 q, 5 q$ and $13 q$ for bicuspid aortic valve and associated cardiovascular malformations. Hum Genet 2007, 121:275-284.

10. Garg V, Muth AN, Ransom JF, Schluterman MK, Barnes R, King IN, Grossfeld PD, Srivastava D: Mutations in NOTCH1 cause aortic valve disease. Nature 2005, 437:270-274.

11. Mohamed SA, Aherrahrou Z, Liptau H, Erasmi AW, Hagemann C, Wrobel S, Borzym K, Schunkert H, Sievers HH, Erdmann J: Novel missense mutations (p.T596M and p.P1797H) in NOTCH1 in patients with bicuspid aortic valve. Biochem Biophys Res Commun 2006, 345:1460-1465.

12. McKellar SH, Tester DJ, Yagubyan M, Majumdar R, Ackerman MJ, Sundt TM 3rd: Novel NOTCH1 mutations in patients with bicuspid aortic valve disease and thoracic aortic aneurysms. J Thorac Cardiovasc Surg 2007, 134:290-296.

13. McBride KL, Riley MF, Zender GA, Fitzgerald-Butt SM, Towbin JA, Belmont JW, Cole SE: NOTCH1 mutations in individuals with left ventricular outflow tract malformations reduce ligand-induced signaling. Hum $\mathrm{Mol}$ Genet 2008, 17:2886-2893.

14. Girdauskas E, Schulz S, Borger MA, Mierzwa M, Kuntze T: Transforming growth factor-beta receptor Type II mutation in a patient with bicuspid aortic valve disease and intraoperative aortic dissection. Ann Thorac Surg 2011, 91:e70-e71.

15. Loscalzo ML, Goh DL, Loeys B, Kent KC, Spevak PJ, Dietz HC: Familial thoracic aortic dilation and bicommissural aortic valve: a prospective analysis of natural history and inheritance. Am J Med Genet A 2007, 143A:1960-1967.

16. Arrington CB, Sower $C T$, Chuckwuk N, Stevens J, Leppert MF, Yetman AT Bowles NE: Absence of TGFBR1 and TGFBR2 mutations in patients with bicuspid aortic valve and aortic dilation. Am J Cardiol 2008, 102:629-631.

17. Laforest $B$, Nemer M: GATA5 interacts with GATA4 and GATA6 in outflow tract development. Dev Biol 2011, 358:368-378.

18. Charron F, Paradis P, Bronchain O, Nemre G, Nemer M: Cooperative interaction between GATA-4 and GATA- 6 regulates myocardial gene expression. Moll Cell Biol 1999, 19:4355. 
19. Laforest B, Andelfinger G, Nemer M: Loss of Gata5 in mice leads to bicuspid aortic valve. J Clin Invest 2011, 121:2876-2887.

20. Padang R, Bagnall RD, Richmond DR, Bannon P, Semsarian C: Rare nonsynonymous variations in the transcriptional activation domains of GATA5 in bicuspid aortic valve disease. J Mol Cell Cardiol 2012, 53:277-281

21. Santiago-Sim T, Mathew-Joseph S, Pannu H, Milewicz DM, Seidman CE, Seidman JG, Kim DH: Sequencing of TGF-beta pathway genes in familial cases of intracranial aneurysm. Stroke 2009, 40:1604-1611.

22. O'Brien KD: Epidemiology and genetics of calcific aortic valve disease. J Investig Med 2007, 55:284-291.

23. Timmerman LA, Grego-Bessa J, Raya A, Bertrán E, Pérez-Pomares JM, Díez J, Aranda S, Palomo S, McCormick F, Izpisúa-Belmonte JC, de la Pompa JL: Notch promotes epithelial-mesenchymal transition during cardiac development and oncogenic transformation. Genes Dev 2004, 18:99-115.

24. Nigam V, Srivastava D: Notch1 represses osteogenic pathways in aortic valve cells. J Mol Cell Cardiol 2009, 47:828-834.

25. O'Brien KD: Pathogenesis of calcific aortic valve disease: a disease process comes of age (and a good deal more). Arterioscler Thromb Vasc Biol 2006, 26:1721-1728.

26. Klüppel M, Wrana JL: Turning it up a Notch: cross-talk between TGF beta and Notch signaling. Bioessays 2005, 27:115-118.

27. El-Hamamsy I, Yacoub MH: Cellular and molecular mechanisms of thoracic aortic aneurysms. Nat Rev Cardiol 2009, 6:771-786.

28. Yetman AT, Graham T: The dilated aorta in patients with congenital cardiac defects. J Am Coll Cardiol 2009, 53:461-467.

29. Kent KC, Crenshaw ML, Goh DL, Dietz HC: Genotype-phenotype correlation in patients with bicuspid aortic valve and aneurysm. $J$ Thorac Cardiovasc Surg 2012. pii: S0022-5223(12)01214-7.

30. NHLBI Exome Sequencing Project (ESP). http://evs.gs.washington.edu/EVS/.

doi:10.1186/1471-2350-14-44

Cite this article as: Foffa et al:: Sequencing of NOTCH1, GATA5, TGFBR1

and TGFBR2 genes in familial cases of bicuspid

aortic valve. BMC Medical Genetics 2013 14:44

\section{Submit your next manuscript to BioMed Central and take full advantage of:}

- Convenient online submission

- Thorough peer review

- No space constraints or color figure charges

- Immediate publication on acceptance

- Inclusion in PubMed, CAS, Scopus and Google Scholar

- Research which is freely available for redistribution 